\title{
Stem/progenitor cell-based transplantation for retinal degeneration: a review of clinical trials
}

\author{
Yiqi Wang ${ }^{1}$, Zhimin Tang ${ }^{1}$ and Ping Gu (1)
}

\begin{abstract}
Retinal degeneration (RD) is one of the dominant causes of irreversible vision impairment and blindness worldwide. However, the current effective therapeutics for RD in the ophthalmologic clinic are unclear and controversial. In recent years, extensively investigated stem/progenitor cells_including retinal progenitor cells (RPCs), embryonic stem cells (ESCs), induced pluripotent stem cells (iPSCs) and mesenchymal stromal cells (MSCs) - with proliferation and multidirectional differentiation potential have presented opportunities to revolutionise the ultimate clinical management of RD. Herein, we provide a comprehensive overview on the progression of clinical trials for RD treatment using four types of stem/progenitor cell-based transplantation to replace degenerative retinal cells and/or to supplement trophic factors from the aspects of safety, effectiveness and their respective advantages and disadvantages. In addition, we also discuss the emerging role of stem cells in the secretion of multifunctional nanoscale exosomes by which stem cells could be further exploited as a potential RD therapy. This review will facilitate the understanding of scientists and clinicians of the enormous promise of stem/progenitor cell-based transplantation for RD treatment, and provide incentive for superior employment of such strategies that may be suitable for treatment of other diseases, such as stroke and ischaemia-reperfusion injury.
\end{abstract}

Facts

- Retinal degeneration (RD) is one of the dominant causes of irreversible vision impairment and blindness worldwide.

- Stem/progenitor cell-based transplantation has been extensively investigated for RD therapy.

- Stem/progenitor cells-mainly including retinal progenitor cells (RPCs), embryonic stem cells (ESCs), induced pluripotent stem cells (iPSCs) and mesenchymal stromal cells (MSCs) - exert effects on sight restoration by replacing and/or rescuing degenerative retinal cells and by secretion of multifunctional nanoscale exosomes.

\footnotetext{
Correspondence: Ping Gu (guping2009@sjtu.edu.cn)

'Department of Ophthalmology, Shanghai Ninth People's Hospital, Shanghai Jiao Tong University School of Medicine, Shanghai Key Laboratory of Orbital Diseases and Ocular Oncology, Shanghai 200011, P.R. China These authors contributed equally: Yiqi Wang, Zhimin Tang Edited by N. Bazan
}

\section{Open questions}

- What are the exact proliferation and differentiation mechanisms of stem/progenitor cells?

- How can the potential tumorigenicity of stem/ progenitor cells and immune rejection caused by exogeneous transplantation strategies be overcome?

- How can rapid clearance of nanovesicle exosomes from tissues or organs be avoided?

\section{Introduction}

Retinal degeneration (RD) is a group of diseases causing blindness via progressive visual loss in humans ${ }^{1}$, and includes age-related macular degeneration $(\mathrm{AMD})^{2}$, diabetic retinopathy $(\mathrm{DR})^{3}$, Stargardt's disease (STGD) ${ }^{4}$ and retinitis pigmentosa $(R P)^{5}$. In particular, AMD is one of the most common ocular diseases clinically, has a global prevalence of $8.7 \%$ with an age of onset varying from 45 to 85 years $^{6}$ and is estimated to affect $\sim 196$ million individuals in western countries by 2020 and 288 million by 
$2040^{7}$. In addition to AMD, DR is also highly prevalent ${ }^{8}$, accounting for $\sim 8.2 \%$ of the global adult population with vision loss ${ }^{9}$. Different from AMD and DR, STGD affects approximately one in 10,000 births $^{10}$, and the total prevalence of different forms of RP varies from one in 2500 to 7000 persons ${ }^{11}$. The human retina is a delicate and elaborate thin sheet composed of ten sublayers ${ }^{12}$, including (1) the inner limiting membrane (ILM), (2) nerve fibre layer (NFL), (3) ganglion cell layer (GCL), (4) inner plexiform layer (IPL), (5) inner nuclear layer (INL), (6) outer plexiform layer (OPL), (7) outer nuclear layer (ONL), (8) outer limiting membrane (OLM), (9) photoreceptor layer (PL) and (10) retinal pigmented epithelium (RPE) monolayer. The photoreceptors play an indispensable role in sensing light signals and visual cues through converting exogenous cues into bioelectrical signals $^{13}$, whereas the RPE cells as a layer of pigment cells transport ions, water and metabolic end products from the subretinal space to the blood, and provide ingested nutrients from the blood to photoreceptors ${ }^{14}$. Although there are differences in pathological progression of various RD diseases, it is currently considered that RPE and/ or photoreceptor dysfunction is the predominate common pathogenesis of $\mathrm{RD}^{15}$, especially when RPE atrophy causes secondary choriocapillaris loss and photoreceptor degeneration, and subsequently results in the detrimental circulatory effects in the dysfunctional RPE and degenerative photoreceptors ${ }^{16}$.

Given the high morbidity of RD threatening all age group burdens of the world, it is urgent to provide effective therapeutic strategies for RD management. Currently, RD patients are routinely recommended to receive medical management, including antioxidants ${ }^{17}$, anti-vascular endothelial growth factor (anti-VEGF) agents $^{18}$, neuroprotective strategies ${ }^{19}$, laser or surgery therapy ${ }^{20}$. Among them, ophthalmologic antioxidant cocktails (e.g., vitamins ${ }^{21}$, lutein and zeaxanthin ${ }^{22}$ ) have been applied to protect retinal cells from oxidative damage, yet the therapeutic outcomes are unsatisfactory due to the unfriendly schedule and underlying biosafety concerns (such as potential risks of skin rashes ${ }^{23}$, haemorrhagic stroke ${ }^{24}$ and lung cancer in cigarette smokers $^{25}$ ). Injection of anti-VEGF agents, including ranibizumab $^{26}$, aflibercept ${ }^{27}$ and bevacizumab ${ }^{28}$, which bind to the VEGF receptors to block VEGF, is mainly used to treat wet $\mathrm{AMD}^{29}$ via inhibition of choroidal neovascularisation ${ }^{30}$. However, adverse reactions of the eyes (such as endophthalmitis, uveitis, retina split holes and vitreous haemorrhage) and systemic adverse reactions (such as hypertension, myocardial infarction and stroke) caused by frequent intravitreal injections and the high cost of treatment lead to poor patient compliance and compromised effectiveness ${ }^{31}$. Neuroprotective interventions are generally divided into two categories ${ }^{19}$-drugs including steroids ${ }^{32}$, dopamine-related therapies ${ }^{33}$ and neurotrophic factors ${ }^{34}$, and rehabilitative methods including physical exercise and electrical stimulation ${ }^{35,36}$; they have been widely used in numerous fundamental studies to slow degenerative progress in the retina by protecting neuronal structure and function ${ }^{19}$, yet their exact clinical efficacy requires further observation and confirmation. Laser therapy is capable of clearing drusen in AMD patients, but may cause inflammatory-related damage and is unable to prevent progression to advanced $\mathrm{AMD}^{37,38}$. Recently, scientists have performed clinical trials involving nanosecond laser treatment for RD, but long-term observation of its safety and effects is still needed $^{39}$. An ideal RD-combating strategy that is generally safe, physiologically stable, highly cost-efficient and targeted at regrowth of retinal cells is appealing and urgently demanded. Fortunately, stem/progenitor cells, including retinal progenitor cells (RPCs), embryonic stem cells (ESCs), induced pluripotent stem cells (iPSCs) and mesenchymal stromal cells (MSCs), are capable of selfrenewal and multidirectional differentiation, and have been extensively studied for biomedical applications to meet the ever-stringent requirements of clinical translation ${ }^{40}$. Numerous well-established preclinical studies of stem/progenitor cell-based therapies in various RD animal models have been conducted by replacing degenerative cells and/or providing nutritional support, and the results suggest great potential in the clinical treatment of $\mathrm{RD}$. More recently, it has been discovered that many types of stem cells, e.g., adipose, bone marrow and umbilical MSCs, can secrete multifunctional exosomes that have low risk of toxicity and immunological rejection, and hold substantial potential for immunotherapy and drug delivery by transmitting numerous biomolecules to specific cells $^{41-44}$. This implies that stem cells will gain additional traction as a promising treatment for $\mathrm{RD}$ due to the advantages of their secreted nanoscale exosomes ${ }^{45}$. A previous review has described in detail the present basic experiments and rationale behind stem/progenitor cellbased transplantation for treatment of $\mathrm{RD}^{46}$. Here, a series of clinical trials based on four stem/progenitor cellsRPCs, ESCs, iPSCs, and MSCs-for RD treatment will be comprehensively reviewed (Table 1), and the underlying working mechanism and the respective advantages and disadvantages of these cells will be discussed in the context of their future clinical application (Scheme 1).

\section{Initial clinical trials based on stem/progenitor cells}

After promising results from studies with a variety of animal models of RD, several initial clinical trials based on RPE or photoreceptors using human tissues or cells were initiated. The pioneering transplantation of foetal human RPE cells into the subretinal space of AMD patients without immunosuppression was initiated by Algvere 


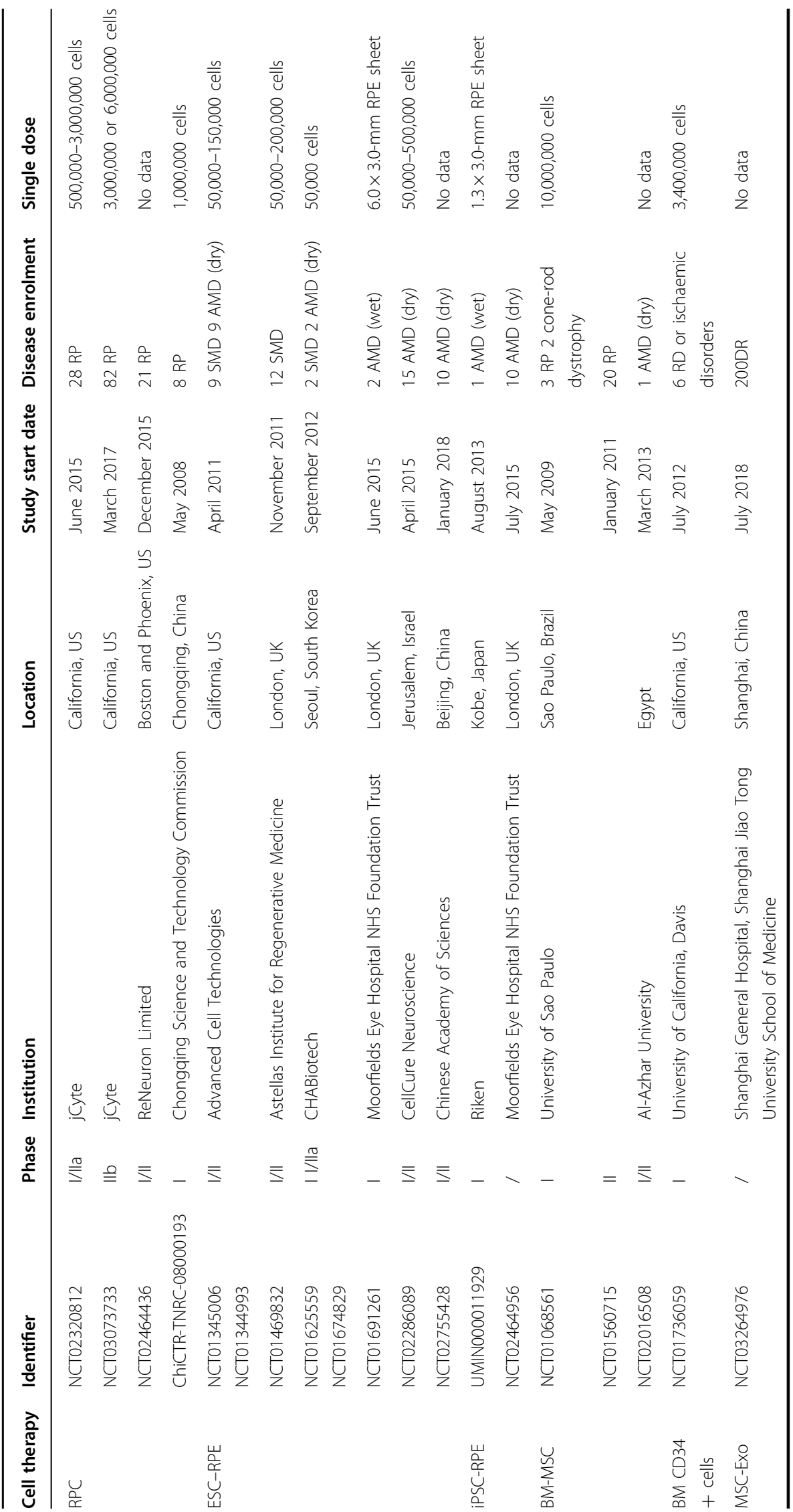




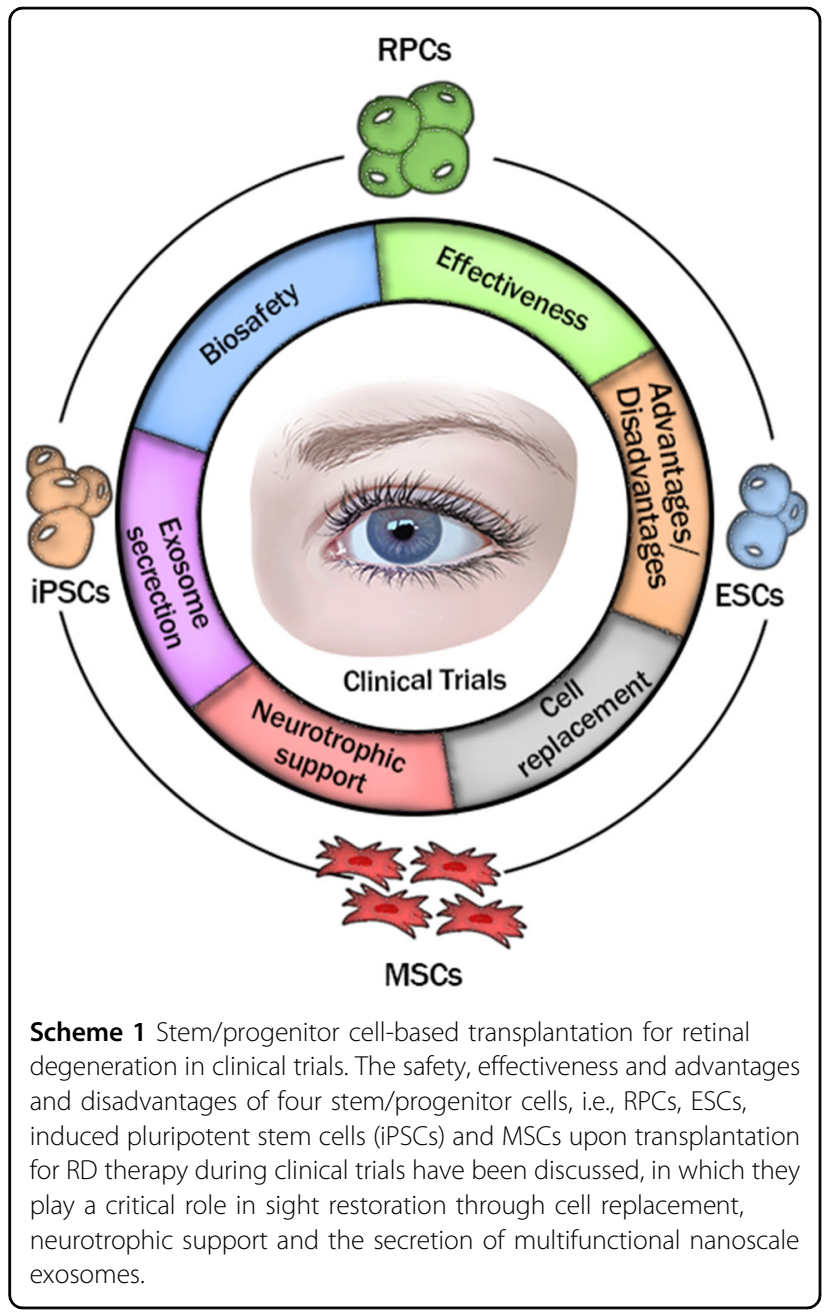

et al. ${ }^{47}$. Host-graft rejection was observed in the exudative lesions over 1-6 months, while little evidence of rejection was detected after 12 months in geographic atrophy of dry AMD, suggesting a possible risk of rejection response in RPE cell transplantation. To further determine the long-term safety of hRPE transplantation in AMD patients, Algvere et al. then carried out another clinical trial by enrolling 16 patients with dry or wet AMD followed by a 2 -year observation ${ }^{48}$. RPE-derived small extrafoveal transplants in the subretinal space of dry AMD patients without immunosuppression did not induce a rejection response, suggesting the potential biosafety of hRPE transplantation in an extrafoveal form for dry AMD treatment. Nevertheless, the improvement in visual acuity demands further confirmation. Almost at the same time, another clinical trial was conducted to establish the biosafety of photoreceptor cell implantation in RP patients. Kaplan et al. transplanted a sheet of photoreceptor cells into the subretinal space of two advanced RP patients without immunosuppression ${ }^{49}$. Twelve months after administration, their visual acuity remained the same (no light perception), and there was no evidence of immune rejection or clinical evidence of detrimental effects, which supports satisfactory biosafety; the effectiveness of cell-based transplantation remains to be exanimated. To further investigate the efficacy of neural retinal transplantation, Humayun et al. conducted a pilot study involving eight advanced RP patients who received human foetal retinal microaggregate suspension and one wet AMD patient who received both human foetal retinal microaggregate suspension and an undissociated retinal sheet ${ }^{50}$. During the first month, three RP patients exhibited transiently improved light sensitivity; however, the effect was reversed in the following months. Although no improvement in light sensitivity was ultimately observed, the study supported the potential effectiveness of cell-based transplanted strategies to some degree. Radtke et al. conducted a similar clinical trial to demonstrate the efficacy of retinal transplantation ${ }^{51}$. Foetal retinal sheets were transplanted subretinally in two RP patients, and threshold objective improvement of visual acuity was observed in one patient 4 months after the surgery. Subsequently, they co-transplanted sheets of foetal neural retina and RPE into the subretinal space of five advanced RP patients, in whom no vision enhancement was observed ${ }^{52}$. Fortunately, seven of ten patients (six RP patients and four AMD patients) experienced favourable outcomes of improved visual acuity in a follow-up clinical trial ${ }^{53}$, providing clinical evidence of the efficacy of cell-based implantation for RD therapy. Although the limited efficacy observed in these early clinical trials based on RPE cells and foetal retinal tissue transplantation did not meet the rigorous demand of commercial clinical translation for RD patients, the technical feasibility of cell-based implantation was preliminarily confirmed so as to support subsequent clinical trials with stem/progenitor cell transplantation.

\section{Stem/progenitor cell-based clinical trials for RD treatment \\ RPCs}

The mature mammalian retina was considered to lack regenerative capacity until the ciliary epithelium was discovered to be the retinal stem cell niche ${ }^{54}$. However, the limited proliferative capacity of pigmented ciliary margin cells in humans impedes their potential use in treating RD. Alternatively, RPCs are a type of neural progenitor cell (NPC) located in the inner layer of the optic $\operatorname{cup}^{55}$. Experiments have shown that in rodents, foetal and postnatal-derived RPCs express several developmental makers (e.g., nestin, Pax6, vimentin, Sox2, Ki$67, \beta$-III tubulin and doublecortin ${ }^{5-58}$ ), suggesting that they possess proliferative capacity similar to that of other stem cells. More importantly, RPCs isolated from various gestational or postnatal periods of rat models can 

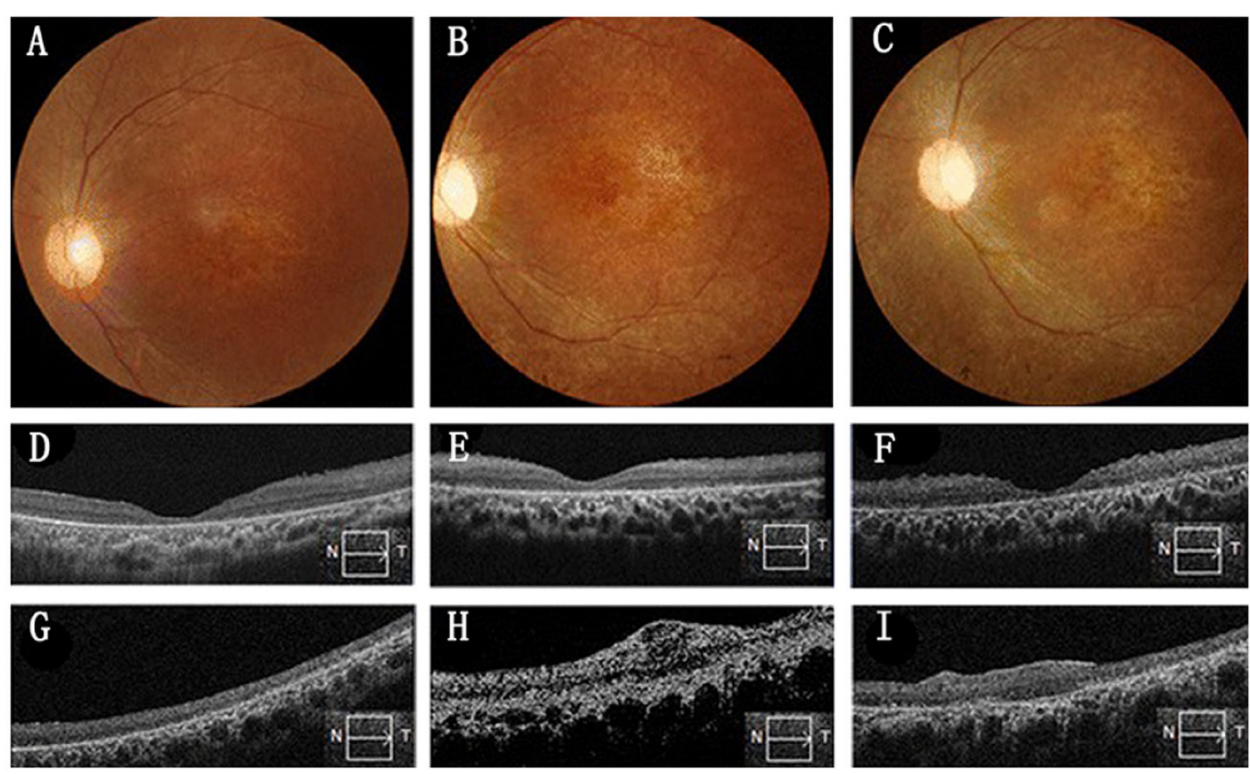

Fig. 1 Colour fundus photographs and OCT images before and after retinal progenitor cell (RPC) transplantation for RP patients. a-c Colour fundus photographs at baseline (a), 12 months after transplantation (b) and 24 months after transplantation (c). No retinal haemorrhage of oedema was observed after transplantation. $\mathbf{d}$-f f Foveal OCT images at baseline (d), 12 months after transplantation (e) and 24 months after transplantation (f). No macular oedema was observed after transplantation. $\mathbf{g - i}$ Horizontal OCT image of the injection site at baseline $(\mathbf{g})$ and after transplantation (h and i). Retinal scarring was observed in this patient $(\mathbf{h}, \mathbf{i})$. Injected RPCs disappeared 24 months after transplantation. Reproduced with permission ${ }^{66}$. Creative Commons Attribution 4.0 International License.

differentiate into various retinal cell types (e.g., bipolar neurons, rod photoreceptors and Müller glial cells ${ }^{59,60}$ ). The specific stem cell properties including proliferation and differentiation displayed by RPCs make RPC transplantation a promising avenue for RD treatment.

\section{Progress of RPC-based clinical trials}

The favourable outcomes in previous fundamental research demonstrated that immature post-mitotic rod precursors used as donor cells can differentiate into rod photoreceptors and integrate into the degenerating retina, thereby improving visual function ${ }^{61}$. On the basis of these observations, scientists further tried to separate foetal tissue-derived RPCs (fRPCs) from the human retina between 14 and 20 weeks of gestation at the time when photoreceptor progenitors are differentiating, and found that these fRPCs can be donor cells for RD treatment $^{62}$. In June 2015, the first FDA-approved Phase I/IIa clinical trial using fRPCs was initiated by Klassen et al. (NCT02320812) ${ }^{63}$. The study enrolled a total of 28 patients with RP, and various doses (0.5-3 million) of foetal tissuederived RPCs were injected into their vitreous cavity as a cell suspension. Twelve months after transplantation, treatmentemergent adverse events (TEAE) were reported in 21 patients, including one patient who suffered from a grade-3 TEAE; improvement of mean best corrected visual acuity (BCVA) in the test eyes varied from 8 to 14 letters without much clinical significance. These outcomes demonstrated the acceptable safety and tolerability of hRPC transplantation that still needs to be improved. A subsequent Phase IIb study designed to evaluate changes in visual function of RP patients following a single injection of hRPCs has completed enrolment (NCT03073733) $^{64}$. Similarly, another FDA-approved Phase I/ II clinical trial (NCT02464436) enrolling 21 RP subjects was conducted at two institutes in Boston and Phoenix ${ }^{65}$. It is a dose-escalation study in which participants with RP received a single subretinal injection of hRPC cells in one eye to evaluate the safety, tolerability and effectiveness of the treatment. This study is still ongoing and is estimated to be completed in July 2021. Aiming to demonstrate the therapeutic effects of RPCbased transplantation, evaluating long-term safety and efficacy is a primary objective of this study. Fortunately, in Asia, Liu et al. evaluated the feasibility and long-term safety of hRPC transplantation in eight advanced RP patients through subretinal injection (ChiCTR-TNRC-08000193) (Fig. 1) ${ }^{66}$. Despite signs of retinal scarring observed after transplantation (Fig. 1h, i), no immunological rejection or tumorigenesis was observed during the 24-month follow-up, which indicated the longterm safety of hRPC transplantation. In addition to improved safety, a significant improvement of BCVA in five eyes and an increase of retinal sensitivity of pupillary response in three patients were also observed between 2 and 6 months after transplantation. However, the improvement did not continue through 12 months. Since the confirmed biosafety and feasibility of hRPC transplantation has laid a solid foundation for vision repair by RPC-based transplantation therapy, the next 

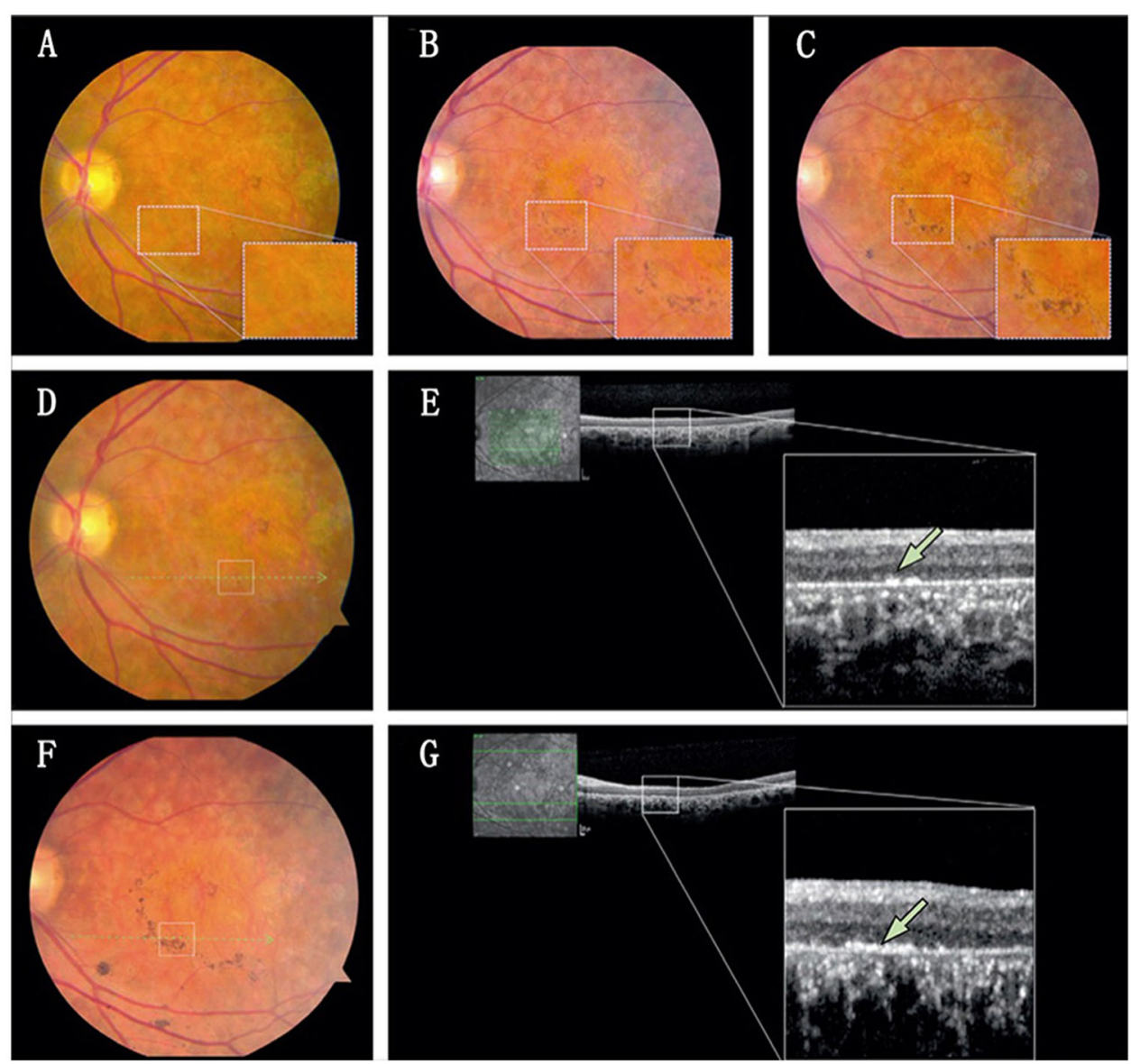

Fig. 2 Colour fundus photographs and OCT images of the left macular in Stargardt's macular dystrophy (SMD) patients before and after hESC-RPE transplantation. a-c Colour fundus photographs at baseline (a), 1 week after transplantation (b) and 6 weeks after transplantation (c). d, $\mathbf{f}$ Macular colour images at baseline (d) and 3 months after transplantation (f). e, $\mathbf{g}$ OCT images at baseline (e) and 3 months after transplantation ( $\mathbf{g}$ ). Colour fundus photographs showed that pigmentation increased continuously from baseline to months 3 , and OCT images showed that it is at the level of RPE. Reproduced with permission ${ }^{74}$. Copyright 2012 Elsevier Ltd.

step is to improve the long-term efficacy of RPC transplantation in RD patients.

\section{Main advantages and disadvantages of RPCS}

The safety and effectiveness of RPC-based transplantation have been widely studied through a series of preclinical and clinical trials. Compared with other stem cells, the main issues facing RPC transplantation are the shortage of sufficient donor cells due to the limited proliferative capacity of $\mathrm{RPCs}^{67}$ and their restricted ability to differentiate into the specific targeted cells ${ }^{68}$. To address these issues, our group found that insulin-like growth factor-1 binds to its receptor and stimulates the PI3K/Akt and MAPK/Erk pathways through increased phosphorylation, resulting in accelerated RPC proliferation ${ }^{69}$. More recently, we synthesised a mussel-inspired injectable hydrogel and its counterpart that are capable of directing RPCs to differentiate towards retinal neurons and promoting proliferation of RPCs ${ }^{70}$. These studies suggest that the shortage of RPCs and limited targeted-differentiation capacity may be resolved. Together with the outstanding advantages of RPCs, such as avoiding ethical issues and the relatively low risk of immune rejection and tumorigenesis, RPCs are considered a good source of donor cells for further clinical RD treatment.

\section{ESCs}

ESCs exhibit limitless proliferation and multidifferentiation into various cell types ${ }^{71}$. The neural progenitors derived from mouse ESCs express regulatory factors to induce retinal differentiation ${ }^{72}$, indicating that ESCs are able to differentiate into the photoreceptor lineage under certain circumstances in vitro and may potentially be an unlimited source for RD treatment.

\section{Progress of ESC-based clinical trials}

Preclinical animal models have shown that ESCs can differentiate into a range of retinal cell types, and several 
clinical trials demonstrated the efficacy and safety of ESC transplantation for the treatment of $\mathrm{RD}^{73}$. In April 2011, Schwartz et al. received the first authorisation from the FDA to initiate a Phase I/II trial involving subretinal transplantation of a low dose of hESC-RPEs (NCT01345006 and NCT01344993) (Fig. 2) ${ }^{74}$. The study enrolled one participant with Stargardt's macular dystrophy (SMD) and one with dry AMD. Four months after transplantation, visual acuity improved from 0 to 5 in the SMD patient and from 21 to 28 in the dry AMD patient, suggesting effectiveness of ESC-based transplantation therapy. It is also encouraging that no adverse proliferation or rejection was detected during the observation period. Since the safety of hESC-RPE transplantation had been demonstrated, in subsequent studies, three dose cohorts $(50,000,100,000$ and 150,000 cells) were implanted into nine SMD participants and nine dry AMD participants $^{75}$. The BCVA in the ten treated eyes and vision-related quality-of-life measures for general and peripheral vision improved 22 months after transplantation with no evidence of serious systemic or ocular adverse reactions. These results supported that hESCRPEs can be donor cells for RD treatment. Encouraged by these outcomes, in November 2011, a more comprehensive investigation of hRPE-ESC transplantation with a higher dose of hESC-RPE cells was conducted in the United Kingdom (NCT01469832) ${ }^{76}$. The investigation enrolled 12 patients suffering from advanced SMD with systemic immunosuppression who received transplantation with one of four doses of hESC-RPE cells $(50,000$, $100,000,150,000$ or 200,000 cells) into the subretinal area. No evidence of uncontrolled proliferation or inflammatory responses was found even after subretinal administration of up to 200,000 cells; however, evidence of benefit at 12 months was also not observed. Although ESC-based transplantation therapy can theoretically improve visual acuity in 12 patients, it was not clinically meaningful due to the severity of retinal degeneration. Even though the ESC transplantation has been conducted for over three decades and has some promising results, there have been no reports on the safety and effectiveness in Asian RD patients. To expand the universality of this treatment, in September 2012, Song et al. initiated the first clinical trial in Asia involving four RD patients (two SMD patients and two dry AMD patients) (NCT01625559 and NCT01674829) by injecting a low a dose of hESC-RPEs $\left(50,000\right.$ cells) per eye ${ }^{77}$. After 12 months of follow-up, three patients exhibited 9-19 letter improvement of visual acuity, which showed that transplantation of hESC-RPE to treat RD is also effective in Asian patients. Since advanced RD ultimately leads to blindness, scientists concentrate on how to improve the visual acuity of these patients. In June 2015, Lyndon et al. performed a Phase I clinical trial with two severe wet AMD patients by delivering an RPE patch containing a hESC-derived RPE monolayer and a basement membrane into the subretinal space $(\text { NCT01691261 })^{78}$. One patient had a 29-letter improvement in visual acuity and one improved by 21 letters after 12 months, which supports the feasibility of RPE patch transplantation in advanced RD treatment. The RPE patch may be a better alternative than cell suspensions. More recently, patients with RD in some clinical trials are being prepared. In Jerusalem (Israel), a Phase I/II clinical trial using OpRegen (a cell-based product composed of RPE cells derived from hESCs) to treat dry AMD (NCT02286089) is still recruiting and is estimated to be completed in December $2024^{79}$. Similarly, in Beijing (China), a Phase I/II clinical trial of subretinal transplantation of hESC-RPEs (NCT02755428) is currently enrolling patients with dry AMD and is estimated to be completed in December $2020^{80}$. In summary, ESCs display an enormous potential for RD by providing millions of target cells required for transplantation.

\section{Main advantages and disadvantages of ESCS}

Compared with harvesting RPCs, it is relatively easy to obtain sufficient ESCs for transplantation. Nevertheless, ESCs have potential for tumour formation due to their high proliferative capacity. Chaudhry et al. integrated both ESCs and ESC-derived neural progenitors into the diseased retinal tissue of rd12 mice, and the proliferation of ESCs eventually resulted in teratoma formation, while ESC-derived neuroprogenitors integrated into the retinal layers ${ }^{81}$. This outcome not only demonstrated the possible tumorigenicity of ESC transplantation, but highlighted that differentiation of ESCs into neuroprogenitors before transplantation may decrease the risk of tumour formation. Accordingly, the existing clinical trials always transplant ESC-derived RPEs into RD patients to reduce the possibility of tumorigenicity. However, ESCs isolated from foetal tissues may be surrounded by ethical concerns $^{73}$, and the multidirectional differentiation also presents difficulties in obtaining the targeted cell types ${ }^{82}$. Moreover, the requirement for lifelong immunosuppressive therapies presents risk and economic burden that further threaten the potential of ESC transplantation ${ }^{83}$. In summary, there are still many challenges to overcome before the clinical use of ESC-based transplantation for $\mathrm{RD}$ therapy.

\section{iPSCs}

In 2006, Takahashi and Yamanaka introduced four factors-Oct3/4, Sox2, c-Myc and Klf4-into mouse embryonic fibroblasts under ES cell culture conditions to induce the iPSC state ${ }^{84}$. The iPSCs were capable of differentiating into all three germ layers after transplantation into foetal and adult mice. One year after the iPSC discovery, they reprogrammed differentiated human somatic 

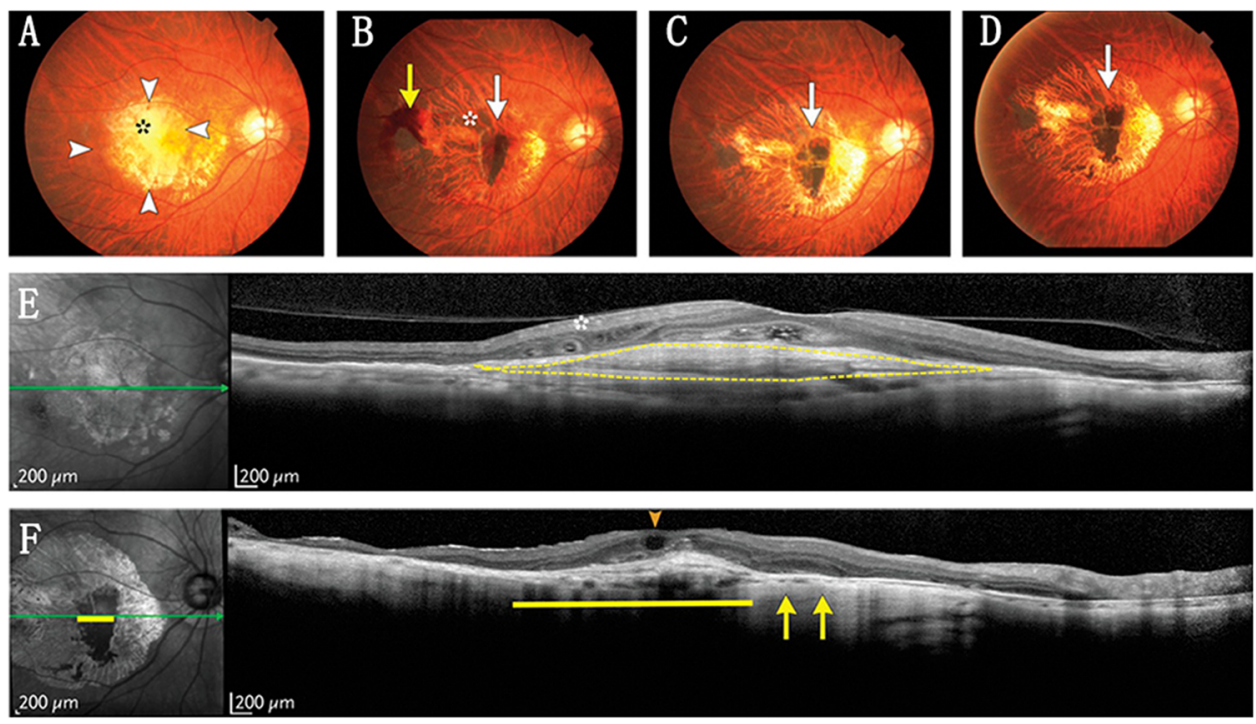

Fig. 3 Colour fundus photographs and OCT images of the right macular in the wet age-related macular degeneration (AMD) patient before and after induced pluripotent stem cell (iPSC)-RPE transplantation. Preoperative and postoperative colour fundus photographs (a-d) and OCT images (e, f) of the iPSC-derived RPE sheet transplantation site in the AMD patient. There were a fibrotic neovascular membrane and polys before transplantation (a). The RPE sheet was curled on day 3 after the surgery (b), but flattened after 8 weeks (c) and lasted until 1 year after transplantation (d). One year after the surgery, the graft sheet could still be observed. OCT images showed that there was a large hyperreflective mass before treatment (e), but it disappeared at postoperative 1 year (f). Reproduced with permission ${ }^{90}$. Copyright 2017 Massachusetts Medical Society.

cells into a pluripotent state with the same four factors ${ }^{85}$. After that, the safety and efficiency of iPSC generation was improved over the last decade. Scientists found that using factors such as valproic acid ${ }^{86}$, SV40 large T antigen ${ }^{87}$ and microRNA $^{88}$ can improve the efficiency of pluripotent induction. Furthermore, the iPSC production safety problem was solved by Nakagawa et al. without the use of $\mathrm{Myc}^{89}$. Since the differentiation potentials of iPSCs and ESCs are similar, scientists have also employed iPSCderived cells to treat RD.

\section{Progress of iPSC-based clinical trials}

The first clinical trial using iPSC-RPE subretinal transplantation was initiated by Takahashi's group (RIKEN in Kobe, Japan) in August 2013 (UMIN000011929) ${ }^{90}$. The study enrolled a 77-year-old Japanese female, who became the first person in the world to receive an autologous iPSC-derived RPE sheet implantation (Fig. 3). One year after transplantation, her vision reduction was stabilised without adverse effects ${ }^{91}$. However, the trial was forced to stop in the subsequent year because of mutations in the second patient's iPSCs and regulatory changes in Japan. Although the investigators declared that mutations are not necessarily tumorigenic, safety issues still need to be reconsidered in human trials, as other scientists have documented genomic instability in iPSCs ${ }^{92}$. To continue the study, Takahashi et al. investigated HLA-matched allogeneic iPSC-derived RPE cells ${ }^{93}$. On 28 March 2017, they enrolled the first 60-year-old Japanese male to receive allogenic iPSC-RPEs in suspension. Compared with autologous iPSCs, the HLA-matched allogeneic iPSCs were safer to administer and more likely to succeed financially. More recently, in Moorfields Eye Hospital in England, an FDA-approved clinical trial of iPSC-RPE subretinal transplantation enrolling ten dry AMD patients is ongoing (NCT02464956) ${ }^{94}$. In the United States, groups of scientists are seeking FDA approval for their clinical studies ${ }^{95}$. Many scientists have aimed to address the safety concerns surrounding iPSC transplantation through the reprogramming process ${ }^{96}$; however, it remains to be seen whether the modifications will work.

\section{Main advantages and disadvantages of iPSCs}

As mentioned above, iPSCs ameliorate the ethical issues of ESCs and have the potential for reduced immunogenicity through autologous transplantation, but iPSCs have a lower variable differentiation efficiency and a relatively high risk of gene mutation ${ }^{97}$. Thus, iPSCs are expected to replace ESC-based therapy in RD treatment.

\section{MSCs}

As a therapeutic option for RD, MSCs mainly provide trophic support via a paracrine mechanism to slow retinal degeneration instead of replacing damaged cells ${ }^{98}$. BMMSCs and ADSCs are two main sources of MSCs with the ability to differentiate into neural retinal cells. Since the feasibility of intravitreal autologous BM-MSC transplantation was demonstrated by Jonas et al. ${ }^{6}$, various clinical 

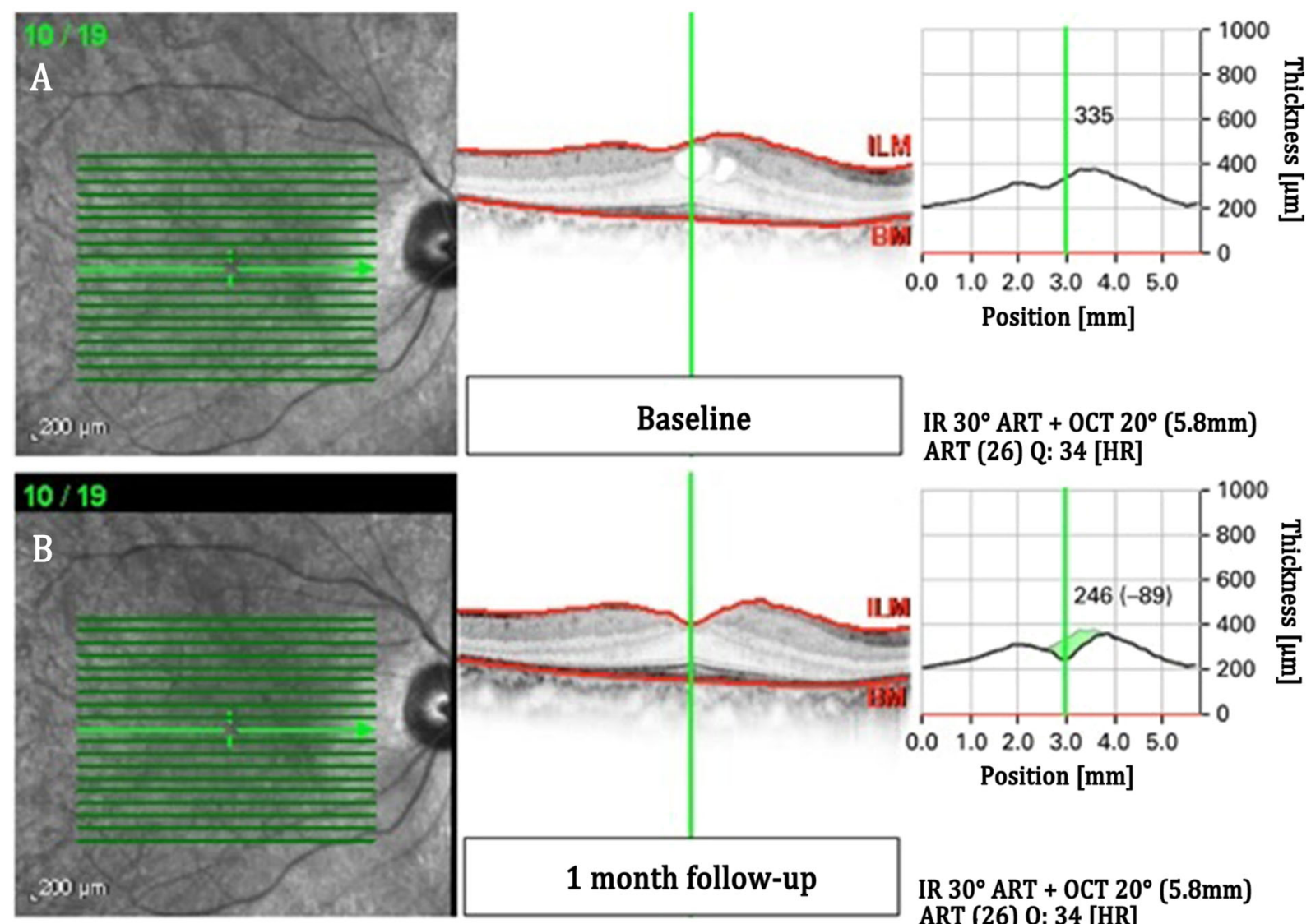
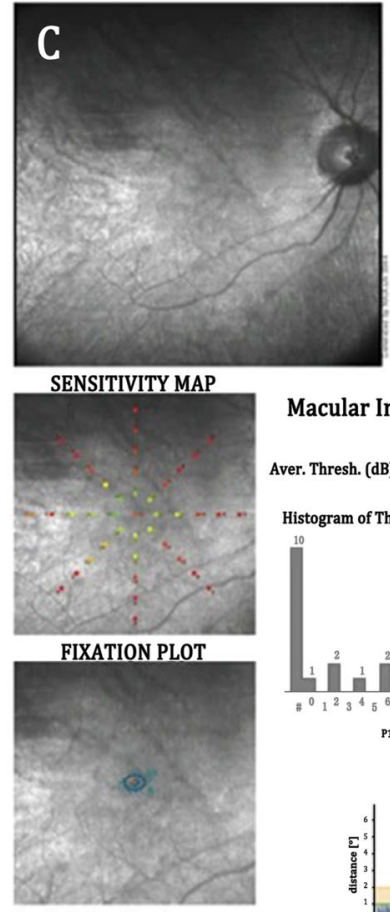

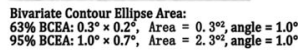

${ }^{10}$

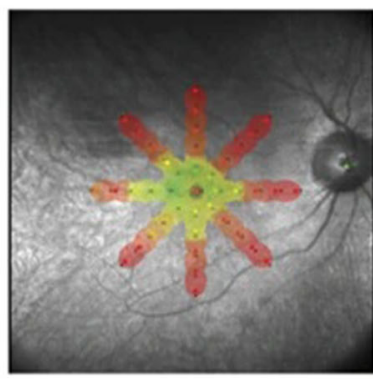

Macular Integrity

Aver. Thresh. (dB)

Histogram of Threshold Frequencies

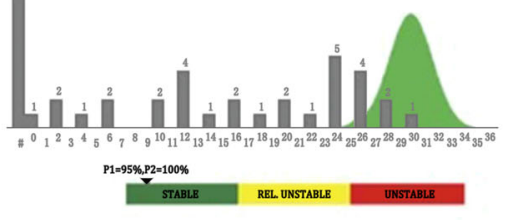

Fixation Graph

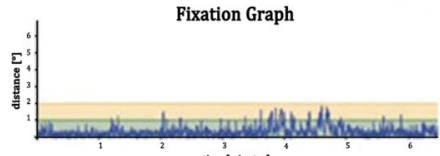

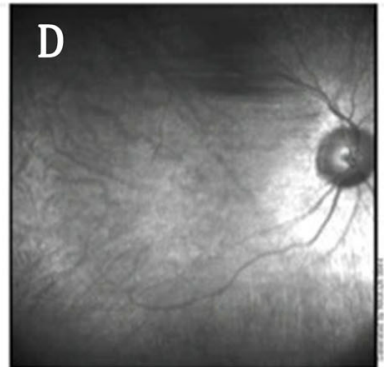

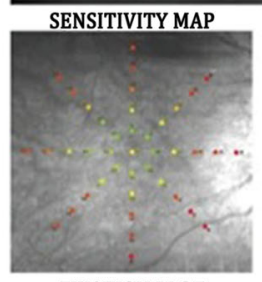

FIXATION PLOT

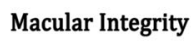

Aver. Thresh. (dB)

Histogram of Threshold Frequencies

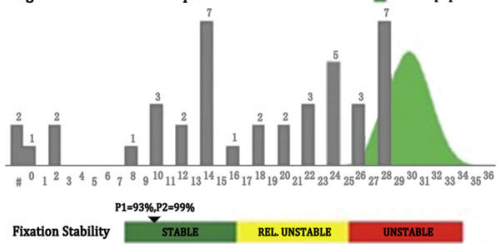

Fixation Graph

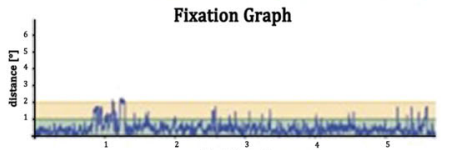

Fig. 4 OCT and microperimetry examinations of RP patient with cystoid macular oedema before and after BM-MSC transplantation. a, b Macular thickness of the patient at baseline (a) and 1 month after the transplantation (b). OCT examinations showed that macular oedema was eliminated after 1-month follow-up. c, d Macular sensitivity of the patient at baseline (c) and 1 month after the transplantation (d). Microperimetry showed that macular sensitivity increased after 1-month follow-up. Reproduced with permission ${ }^{102}$. Copyright 2012 Springer Nature. 
trials of BM-MSC transplantation have been carried out ${ }^{99}$. Studies have demonstrated that BM-MSCs and ADSCs share similar immunomodulatory capacities ${ }^{100}$. Therefore, ADSCs are considered an alternative to BM-MSCs for RD treatment, despite the few current studies on ADSC-based therapy.

\section{Progress of MSC-based clinical trials}

The progress of BM-MSC transplantation therapy has been accelerated towards the clinic. In May 2009, Siqueira et al. initiated a Phase I trial in three patients with RP and two patients with cone-rod dystrophy by injecting autologous BM-MSCs into the vitreous cavity (NCT01068561) (Fig. 4) ${ }^{101,102}$. Ten months after transplantation, there was no detectable structural or functional toxicity, demonstrating the short-term safety of the transplantation. Based on the promising result, a Phase II study was initiated in June 2011 to further confirm the efficacy of BM-based transplantation therapy (NCT01560715) ${ }^{103}$. The study enrolled $20 \mathrm{RD}$ patients who received a vitreous implantation of BM-MSCs, finding that vision-related life quality improved 3 months post treatment, supporting the potential efficacy of BM-MSC therapy; however, it was transitory and no longer evident at 12 months post treatment. Similarly, 1 year later, another pilot clinical study was initiated by Park et al. by injecting autologous CD34 $4^{+}$BM-MSCs into the vitreous cavity of six patients with retinal vascular occlusion or RD (NCT01736059) ${ }^{104}$. The study is ongoing, and preliminary findings from the Phase I patients (six patients enrolled between November 2012 and August 2014) have been published. Within 6 months of follow-up, the autologous cells appear to be well tolerated, yet efficacy still requires further exploration. These unsatisfactory results may be due to the mechanism of BM-based transplantation therapy, namely, the neurotrophic effect that can only support the survival of photoreceptors instead of promoting their regeneration. More recently, an ongoing BM-MSC-based therapy for RD in Saudi Arabi was registered online $\left(\right.$ NCT02016508) ${ }^{105}$. There are increasingly safety concerns surrounding intravitreal administration of autologous BM-MSCs. Satarian et al. conducted a Phase I clinical trial to examine the safety of autologous BM-MSC transplantation. Three patients with advanced RP were enrolled and received intravitreal injection of autologous BM-MSCs ${ }^{106}$. After 2 years of follow-up, severe fibrous tissue proliferation was observed in the injection site of the third patient, leading to iris neovascularisation, formation of mature cataracts and tractional retinal detachment. Both the loss of improvement over time and the existing safety concerns may imply that BM-MSCs are not the best choice for RD treatment.

\section{Main advantages and disadvantages of MSCs}

To date, scientists have shown the neurotrophic effect of MSCs for treating $\mathrm{RD}^{107}$; however, little evidence of cellular replacement has been established. The cytokines $^{108}$, growth factors ${ }^{99,109}$, micro- and nanolipid macrovesicles $^{110}$ secreted by MSCs exert important angiogenic, immunomodulatory, anti-apoptotic and antiinflammatory effects that provide trophic support for the degenerative retina. Further, the neurotropic effect cannot solve the intrinsic problem of RD, which is the fatal flaw of MSC-based transplantation therapy. In addition, adverse effects, e.g., iris neovascularisation observed in the eye receiving autologous MSC cell transplantation for RD therapy, may be partially caused by the lack of quality control of MSCs injected as autologous transplants and the high variability in MSCs derived from different individuals $^{106}$, thus raising safety concerns of MSC-based transplantation therapy for RD. Moreover, the potential heterogeneous nature of MSCs restricts them from expanding into specific cells such as BM CD34+cells, which may also be an issue for MSCs ${ }^{111}$. Last but not least, MSCs primarily differentiate into mesodermalderived tissues far from the targeted retinal cells, and their regenerative potential declines with increasing donor age $^{112}$. This further limits their promise as an RD treatment even if MSCs demonstrate immunosuppressive effects and are less immunogenic than other stem cells $^{113,114}$.

\section{Stem cell-derived exosomes for RD treatment}

In addition to replacement and neurotrophic mechanisms based on stem/progenitor cells, the latest studies have focused on extracellular exosomes secreted by stem cells as a potential therapy for RD. Several studies have identified that some types of MSCs secrete exosomes (MSC-Exo), mainly adipose, bone marrow and umbilical $\mathrm{MSCs}^{45}$. Exosomes are cell-derived nanovesicles that have low toxicity, low risk of immunological rejection, exquisite target-homing specificity and potential for drug/gene delivery ${ }^{115}$. Accordingly, exosome-based therapy is gaining research attention for multiple diseases throughout the body ${ }^{45}$. For instance, scientists have found that MSCExo has a prominent therapeutic effect in central nervous system diseases, such as stroke ${ }^{116,117}$, Alzheimer's disease $^{118}$ and spinal cord injury ${ }^{119}$. Importantly, the positive roles of MSC-Exo in anatomical and functional restoration of ocular tissues, e.g., cornea, optic nerve and retina, have also been confirmed in several types of eye diseases, including optic nerve crush $^{42}$, glaucoma ${ }^{120}$, retinal ischaemia ${ }^{121}$ and $\mathrm{DR}^{122}$, by modulating angiogenesis and inflammation pathways, immunomodulation or even tissue regeneration. As reported, Safwat et al. transplanted rabbit adipose MSC-Exo to the eyes of rabbits with 
diabetes mellitus (DM) by subconjunctival and intravitreal injection. They found that the implanted MSC-Exo efficiently delivered miRNA-222 into the retina to prevent the progression of retinal degeneration ${ }^{122}$. Similarly, Zhang et al. discovered that human umbilical cord MSCExo reduces hyperglycaemia-induced retinal inflammation after intravitreal injection into rabbis with $\mathrm{DM}^{123}$. Based on the high efficacy of MSC-Exo for RD control in preclinical trials, a clinical trial intending to evaluate the function of serum exosomal miRNA in the pathogenesis of DR has obtained FDA approval (NCT03264976) and will soon recruit patients ${ }^{124}$. Stem cell-derived exosomes may play an important role in RD treatment in the future.

\section{Conclusions}

$\mathrm{RD}$ is a leading cause of blindness worldwide, and it mainly results from the degeneration of RPE and photoreceptor cells. Accordingly, scientists have applied progenitor/stem cell-based transplantation therapy to RD treatment, especially the use of RPC-, ESC-, iPSC- and MSC-based therapies, by replacing degenerative retinal cells and/or preventing retinal degeneration by supplementing trophic factors. Most importantly, stem cells also secrete multifunctional exosomes and serve as pathogenic mediators between cells in the eyes, which are essential working mechanisms underlying how stem cell-based therapy impacts RD. Through thorough discussion on the advantages and disadvantages of different kinds of stem/ progenitor cells, we hypothesise that RPCs are the best candidates for RD treatment since they do not present ethnical concerns and they have a relatively low risk of rejection and tumorigenesis. Although there are some limitations in RPC proliferation and differentiation using current technologies, emerging culture techniques (as described above) will provide an opportunity to solve the problems. In addition, to increase the safety and efficacy of stem/progenitor cell-based transplantation therapy, several unsolved issues and corresponding strategies need to be resolved:

(1) Currently, research on stem cell-derived exosomebased strategies for biomedical applications is still in its infancy. One of the main weaknesses of nanovesicle exosomes is their rapid clearance from tissues or organs. Clearance should be verified in ophthalmology after topical, intravitreal or subconjunctival applications to determine how to overcome this issue. On the other hand, exosomes as a sustained delivery platform greatly rely on the generation of vesicles of consistently high purity and quality on a large scale, which is another challenge. Hence, more methods and technologies for exosome-based systems should be developed to generate the next stem cell-derived exosome nanomedicine for RD management.
(2) The proliferation and differentiation mechanisms of stem/progenitor cells still require better understanding. Thus, besides clinical trials, more basic experiments need to be conducted for a deeper comprehension of the mechanism, which will facilitate the application of progenitor/stem cell-based transplantation therapy in more RD patients as early as possible.

(3) Potential tumorigenicity of stem cells and immune rejection caused by exogenous transplantation strategies does not meet the clinical safety threshold. Fortunately, fundamental experiments have shown that preinduction of ESCs into neural progenitors before transplantation may reduce tumorigenicity ${ }^{81}$, providing a feasible solution to this problem. Meanwhile, immunosuppressive therapies also evolve, and new techniques such as the xeno-free techniques are being developed to reduce the immune response. Thus, the issue of immune rejection is expected to be addressed in the future.

(4) Ethical issues of stem cell transplantation need to be resolved. On one hand, iPSC generation does not present the same ethical concerns as ESC harvesting. On the other hand, iPSC-based strategy is a completely new field and remains in its infancy; experts will reach an ethical consensus over time.

(5) Most of the current clinical trials are in the early I/IIa phases. Thus, there is still a long way to go before their findings can be applied to clinical practice.

All in all, with deepening research, stem/progenitor cellbased transplantation will be an essential treatment used in the clinic that will bring new hope to RD patients through the joint efforts of doctors and researchers.

\section{Acknowledgements}

The research was supported by National Natural Science Foundations of China (No. 81870687), Key R\&D program of China (No. 2018YFC1106100), Key program of Shanghai Science and Technology Commission (No. 19JC1415503), Shanghai Municipal Education Commission-Gaofeng Clinical Medicine Grant Support (No. 20161316) and Science and Technology Commission of Shanghai (No. 17DZ2260100).

\section{Conflict of interest}

The authors declare that they have no conflict of interest.

\section{Publisher's note}

Springer Nature remains neutral with regard to jurisdictional claims in published maps and institutional affiliations.

Received: 13 May 2020 Revised: 21 August 2020 Accepted: 27 August 2020 Published online: 23 September 2020

\footnotetext{
References

1. Dalkara, D., Goureau, O., Marazova, K. \& Sahel, J. A. Let there be light: gene and cell therapy for blindness. Hum. Gene Ther. 27, 134-147 (2016).

2. Jonas, J. B. Global prevalence of age-related macular degeneration. Lancet Glob. Health 2, e65-e66 (2014)
} 
3. Liu, H. et al. Photoreceptor cells influence retinal vascular degeneration in mouse models of retinal degeneration and diabetes. Investig. Ophthalmol. Vis. Sci. 57, 4272-4281 (2016).

4. Xin, W. et al. Identification of genetic defects in 33 probands with stargardt disease by wes-based bioinformatics gene panel analysis. PLOS ONE 10, e0132635 (2015)

5. Sorrentino, F. S., Gallenga, C. E., Bonifazzi, C. \& Perri, P. A challenge to the striking genotypic heterogeneity of retinitis pigmentosa: a better understanding of the pathophysiology using the newest genetic strategies. Eye $\mathbf{3 0}$ 1542-1548 (2016).

6. Jonas, J. B., Cheung, C. M. G. \& Panda-Jonas, S. Updates on the epidemiology of age-related macular degeneration. Asia-Pac. J. Ophthalmol. 6, 493-497 (2017).

7. Wong, W. L. et al. Global prevalence of age-related macular degeneration and disease burden projection for 2020 and 2040: a systematic review and meta-analysis. Lancet Glob. Health 2, e106-e116 (2014).

8. Ting, D. S. W., Cheung, G. C. M. \& Wong, T. Y. Diabetic retinopathy: global prevalence, major risk factors, screening practices and public health challenges: a review. Clin. Exp. Ophthalmol. 44, 260-277 (2016).

9. Raman, R., Gella, L., Srinivasan, S. \& Sharma, T. Diabetic retinopathy: an epidemic at home and around the world. Indian J. Ophthalmol. 64, 69-75 (2016).

10. Tsang, S. H. \& Sharma, T. Stargardt disease. Adv. Exp. Med. Biol. 1085, 139-151 (2018)

11. Parmeggiani, F. Clinics, epidemiology and genetics of retinitis pigmentosa Curr. genomics 12, 236-237 (2011).

12. Grossniklaus, H. E., Geisert, E. E. \& Nickerson, J. M. Introduction to the retina Prog. Mol. Biol. Transl. Sci. 134, 383-396 (2015).

13. Gollisch, T. \& Meister, M. Eye smarter than scientists believed: neural computations in circuits of the retina. Neuron 65, 150-164 (2010).

14. Ma, X. et al. The transcription factor MITF in RPE function and dysfunction Prog. Retin Eye Res. 73, 100766 (2019).

15. Ao, J., Wood, J. P., Chidlow, G., Gillies, M. C. \& Casson, R. J. Retinal pigment epithelium in the pathogenesis of age-related macular degeneration and photobiomodulation as a potential therapy? Clin. Exp. Ophthalmol. 46 670-686 (2018).

16. DeRamus, M. L. et al. Selective ablation of dehydrodolichyl diphosphate synthase in murine retinal pigment epithelium (RPE) causes RPE atrophy and retinal degeneration. Cells 9, 771 (2020).

17. Evans, J. R. \& Lawrenson, J. G. Antioxidant vitamin and mineral supplements for preventing age-related macular degeneration. Cochrane Database Syst. Rev. 7, CD000253-CD000253 (2017).

18. Bakri, S. J. et al. Safety and efficacy of anti-vascular endothelial growth factor therapies for neovascular age-related macular degeneration: a report by the American Academy of Ophthalmology. Ophthalmology 126, 55-63 (2019).

19. Pardue, M. T. \& Allen, R. S. Neuroprotective strategies for retinal disease. Prog. Retin Eye Res. 65, 50-76 (2018).

20. Findlay, Q. et al. Prophylactic laser in age-related macular degeneration: the past, the present and the future. Eye 32, 972-980 (2018).

21. Merle, B. M. J., Silver, R. E., Rosner, B. \& Seddon, J. M. Dietary folate, B vitamins, genetic susceptibility and progression to advanced nonexudative agerelated macular degeneration with geographic atrophy: a prospective cohort study. Am. J. Clin. Nutr. 103, 1135-1144 (2016).

22. Eisenhauer, B., Natoli, S., Liew, G. \& Flood, V. M. Lutein and zeaxanthin-food sources, bioavailability and dietary variety in age-related macular degeneration protection. Nutrients $\mathbf{9}, 120$ (2017).

23. Gaziano, J. M. et al. Multivitamins in the prevention of cancer in men: the Physicians' Health Study II randomized controlled trial. J. Am. Med. Assoc. 308, 1871-1880 (2012).

24. Le, N. K., Kesayan, T., Chang, J. Y. \& Rose, D. Z. Z. Cryptogenic intracrania hemorrhagic strokes associated with hypervitaminosis $\mathrm{E}$ and acutely elevated a-tocopherol levels. J. Stroke Cerebrovasc. Dis. 29, 104747 (2020).

25. Alsharairi, N. A. The effects of dietary supplements on asthma and lung cancer risk in smokers and non-smokers: a review of the literature. Nutrients 11, 725 (2019)

26. Chong, V. Ranibizumab for the treatment of wet AMD: a summary of realworld studies. Eye 30, 270-286 (2016).

27. Haga, A., Kawaji, T., Ideta, R., Inomata, Y. \& Tanihara, H. Treat-and-extend versus every-other-month regimens with aflibercept in age-related macular degeneration. Acta Ophthalmol. 96, e393-e398 (2018).
28. Vo, T. A., Abedi, S., Schneider, K., Chwa, M. \& Kenney, M. C. Effects of bevacizumab, ranibizumab, and aflibercept on phagocytic properties in human RPE cybrids with AMD versus normal mitochondria. Exp. Eye Res. 177 112-116 (2018).

29. Khanna, S. et al. Current and upcoming anti-VEGF therapies and dosing strategies for the treatment of neovascular AMD: a comparative review. BMJ Open Ophthalmol. 4, e000398 (2019).

30. Hachana, S. et al. The effects of anti-VEGF and kinin B(1) receptor blockade on retinal inflammation in laser-induced choroidal neovascularization. Br. J. Pharmacol. 177, 1949-1966 (2019).

31. van Asten, F. et al. The cost-effectiveness of bevacizumab, ranibizumab and aflibercept for the treatment of age-related macular degeneration-a costeffectiveness analysis from a societal perspective. PLOS ONE 13, e0197670-e0197670 (2018)

32. Allen, R. S. et al. Progesterone treatment shows greater protection in brain vs. retina in a rat model of middle cerebral artery occlusion: progesterone receptor levels may play an important role. Restor. Neurol. Neurosci. 34, 947-963 (2016).

33. Brilliant, M. H. et al. Mining retrospective data for virtual prospective drug repurposing: L-DOPA and age-related macular degeneration. Am. J. Med. 129, 292-298 (2016).

34. Önger, M. E. et al. The role of growth factors in nerve regeneration. Drug Discov. Ther. 10, 285-291 (2017).

35. Lawson, E. C. et al. Aerobic exercise protects retinal function and structure from light-induced retinal degeneration. J. Neurosci. 34, 2406-2412 (2014).

36. Morimoto, T. et al. Characteristics of retinal reflectance changes induced by transcorneal electrical stimulation in cat eyes. PLOS ONE 9, e92186-e92186 (2014).

37. Han, J. W. et al. Comparison of the neuroinflammatory responses to selective retina therapy and continuous-wave laser photocoagulation in mouse eyes. Graefe's Arch. Clin. Exp. Ophthalmol. = Albrecht von. Graefes Arch. fur klinische und experimentelle Ophthalmologie 256, 341-353 (2018).

38. Guymer, R. H. et al. Subthreshold nanosecond laser intervention in agerelated macular degeneration: the LEAD randomized controlled clinical trial. Ophthalmology 126, 829-838 (2019).

39. Lek, J. J. et al. Subthreshold nanosecond laser intervention in intermediate age-related macular degeneration: study design and baseline characteristics of the laser in early stages of age-related macular degeneration study (report number 1). Ophthalmol. Retin. 1, 227-239 (2017).

40. Tang, Z. et al. Progress of stem/progenitor cell-based therapy for retinal degeneration. J. Transl. Med. 15, 99 (2017).

41. Yu, B. et al. Exosomes derived from MSCs ameliorate retinal laser injury partially by inhibition of MCP-1. Sci. Rep. 6, 34562 (2016).

42. Mead, B. \& Tomarev, S. Bone marrow-derived mesenchymal stem cellsderived exosomes promote survival of retinal ganglion cells through miRNAdependent mechanisms. Stem Cells Transl. Med. 6, 1273-1285 (2017).

43. Zhang, X. et al. Effects of mesenchymal stem cells and their exosomes on the healing of large and refractory macular holes. Graefe's Arch. Clin. Exp. Ophthalmol. = Albrecht von. Graefes Arch. fur klinische und experimentelle Ophthalmologie 256, 2041-2052 (2018).

44. Samaeekia, R. et al. Effect of human corneal mesenchymal stromal cellderived exosomes on corneal epithelial wound healing. Investig. Ophthalmol. Vis. Sci. 59, 5194-5200 (2018).

45. Mead, B. \& Tomarev, S. Extracellular vesicle therapy for retinal diseases. Prog. Retin Eye Res. 100849 (2020)

46. Singh, M. S. et al. Retinal stem cell transplantation: balancing safety and potential. Prog. Retin Eye Res 75, 100779 (2020).

47. Algvere, P. V., Berglin, L., Gouras, P., Sheng, Y. \& Kopp, E. D. Transplantation of RPE in age-related macular degeneration: observations in disciform lesions and dry RPE atrophy. Graefe's Arch. Clin. Exp. Ophthalmol. = Albrecht von. Graefes Arch. fur klinische und experimentelle Ophthalmologie 235, 149-158 (1997).

48. Algvere, P. V., Gouras, P. \& Dafgård Kopp, E. Long-term outcome of RPE allografts in non-immunosuppressed patients with AMD. Eur. J. Ophthalmol. 9, 217-230 (1999).

49. Kaplan, H. J., Tezel, T. H., Berger, A. S., Wolf, M. L. \& Del Priore, L. V. Human photoreceptor transplantation in retinitis pigmentosa. A safety study. Arch Ophthalmol. 115, 1168-1172 (1997).

50. Humayun, M. S. et al. Human neural retinal transplantation. Investig. Ophthalmol. Vis. Sci. 41, 3100-3106 (2000). 
51. Radtke, N. D., Aramant, R. B., Seiler, M. \& Petry, H. M. Preliminary report: indications of improved visual function after retinal sheet transplantation in retinitis pigmentosa patients. Am. J. Ophthalmol. 128, 384-387 (1999).

52. Radtke, N. D., Seiler, M. J., Aramant, R. B., Petry, H. M. \& Pidwell, D. J. Transplantation of intact sheets of fetal neural retina with its retinal pigment epithelium in retinitis pigmentosa patients. Am. J. Ophthalmol. 133, 544-550 (2002)

53. Radtke, N. D. et al. Vision improvement in retinal degeneration patients by implantation of retina together with retinal pigment epithelium. Am. J. Ophthalmol. 146, 172-182 (2008).

54. Tropepe, $\mathrm{V}$. et al. Retinal stem cells in the adult mammalian eye. Science $\mathbf{2 8 7}$ 2032-2036 (2000).

55. Prada, C., Puga, J., Pérez-Méndez, L., López, R. \& Ramírez, G. Spatial and temporal patterns of neurogenesis in the chick retina. Eur. J. Neurosci. $\mathbf{3}$ 559-569 (1991).

56. Klassen, $H$. J. et al. Multipotent retinal progenitors express developmental markers, differentiate into retinal neurons, and preserve light-mediated behavior. Investig. Ophthalmol. Vis. Sci. 45, 4167-4173 (2004).

57. Semo, M. et al. Efficacy and safety of human retinal progenitor cells. Transl. Vis. Sci. Technol. 5, 6 (2016).

58. Remez, L. A. et al. Pax6 is essential for the generation of late-born retinal neurons and for inhibition of photoreceptor-fate during late stages of retinogenesis. Dev. Biol. 432, 140-150 (2017).

59. Turner, D. L. \& Cepko, C. L. A common progenitor for neurons and glia persists in rat retina late in development. Nature 328, 131-136 (1987).

60. Qiu, G. et al. Revisiting nestin expression in retinal progenitor cells in vitro and after transplantation in vivo. Exp. Eye Res. 84, 1047-1059 (2007).

61. MacLaren, R. E. et al. Retinal repair by transplantation of photoreceptor precursors. Nature 444, 203-207 (2006).

62. Hendrickson, A. et al. Rod photoreceptor differentiation in fetal and infant human retina. Exp. Eye Res. 87, 415-426 (2008).

63. U.S. jCyte. Safety of a single, intravitreal injection of human retinal progenitor cells (jCell) in retinitis pigmentosa. https:/clinicaltrials.gov/ct2/show/study/ NCT02320812. Accessed July 21, 2020 (2020)

64. U.S. jCyte. Safety and efficacy of intravitreal injection of human retinal progenitor cells in adults with retinitis pigmentosa. https:/clinicaltrials.gov/ct2/ show/study/NCT03073733. Accessed July 21, 2020 (2020).

65. U.S. ReNeuron Limited. Safety and tolerability of hPRC in retinitis pigmentosa (hRPCRP). https://clinicaltrials.gov/ct2/show/study/NCT02464436. Accessed July 21, 2020 (2020).

66. Liu, Y. et al. Long-term safety of human retinal progenitor cell transplantation in retinitis pigmentosa patients. Stem Cell Res. Ther. 8, 209 (2017).

67. Ballios, B. G. et al. A hyaluronan-based injectable hydrogel improves the survival and integration of stem cell progeny following transplantation. Stem Cell Rep. 4, 1031-1045 (2015).

68. Gu, P. et al. Isolation of retinal progenitor and stem cells from the porcine eye. Mol. Vis. 13, 1045-1057 (2007).

69. Wang, Y. et al. Insulin-like growth factor-1 regulation of retinal progenitor cell proliferation and differentiation. Cell Cycle 17, 515-526 (2018).

70. Tang, Z. et al. Mussel-inspired injectable hydrogel and its counterpart for actuating proliferation and neuronal differentiation of retinal progenitor cells. Biomaterials 194, 57-72 (2019).

71. Jia, J. et al. Regulation of pluripotency and self- renewal of ESCs through epigenetic-threshold modulation and mRNA pruning. Cell 151, 576-589 (2012).

72. Singh, R. K. et al. Characterization of three-dimensional retinal tissue derived from human embryonic stem cells in adherent monolayer cultures. Stem Cells Dev. 24, 2778-2795 (2015).

73. Shenfield, F. Semantics and ethics of human embryonic stem-cell research. Lancet 365, 2071-2073 (2005)

74. Schwartz, S. D. et al. Embryonic stem cell trials for macular degeneration: a preliminary report. Lancet 379, 713-720 (2012).

75. Schwartz, S. D. et al. Human embryonic stem cell-derived retinal pigment epithelium in patients with age-related macular degeneration and Stargardt's macular dystrophy: follow-up of two open-label phase 1/2 studies. Lancet 385, 509-516 (2015).

76. Mehat, M. S. et al. Transplantation of human embryonic stem cell-derived retinal pigment epithelial cells in macular degeneration. Ophthalmology $\mathbf{1 2 5}$ 1765-1775 (2018).
77. Song, W. K. et al. Treatment of macular degeneration using embryonic stem cell-derived retinal pigment epithelium: preliminary results in Asian patients. Stem Cell Rep. 4, 860-872 (2015).

78. da Cruz, L. et al. Phase 1 clinical study of an embryonic stem cell-derived retinal pigment epithelium patch in age-related macular degeneration. Nat. Biotechnol. 36, 328-337 (2018).

79. Israel. CellCure Neurosciense. Safety and efficacy study of OpRegen for treatment of advanced dry-form age-related macular degeneration. https:// clinicaltrials.gov/ct2/show/study/NCT02286089. Accessed July 21, 2020 (2020).

80. China. Chinese Academy of Science. Subretinal transplantation of retinal pigment epitheliums in treatment of age-related macular degeneration diseases. https:/clinicaltrials.gov/ct2/show/study/NCT02755428. Accessed July 21, 2020 (2020).

81. Chaudhry, G. R. et al. Fate of embryonic stem cell derivatives implanted into the vitreous of a slow retinal degenerative mouse model. Stem Cells Dev. 18, 247-258 (2009).

82. Vugler, A. et al. Elucidating the phenomenon of HESC-derived RPE: anatomy of cell genesis, expansion and retinal transplantation. Exp. Neurol. 214, 347-361 (2008).

83. Allison, T. L. Immunosuppressive therapy in transplantation. Nurs. Clin. North Am. 51, 107-120 (2016).

84. Takahashi, K. \& Yamanaka, S. Induction of pluripotent stem cells from mouse embryonic and adult fibroblast cultures by defined factors. Cell 126, 663-676 (2006)

85. Takahashi, K. Induction of pluripotent stem cells from adult human fibroblasts by defined factors. Cell 131, 861-872 (2007).

86. Zhai, Y. et al. Histone deacetylase inhibitor valproic acid promotes the induction of pluripotency in mouse fibroblasts by suppressing reprogramming-induced senescence stress. Exp. Cell Res. 337, 61-67 (2015).

87. Tan, L. et al. Naked mole rat cells have a stable epigenome that resists iPSC reprogramming. Stem Cell Rep. 9, 1721-1734 (2017).

88. Zhang, L. et al. MiR-134-Mbd3 axis regulates the induction of pluripotency. J. Cell. Mol. Med. 20, 1150-1158 (2016)

89. Nakagawa, M. et al. Generation of induced pluripotent stem cells without Myc from mouse and human fibroblasts. Nat. Biotechnol. 26, 101-106 (2008).

90. Mandai, M. et al. Autologous induced stem-cell-derived retinal cells for macular degeneration. N. Engl. J. Med. 376, 1038-1046 (2017).

91. Mandai, M., Kurimoto, Y. \& Takahashi, M. Autologous induced stem-cellderived retinal cells for macular degeneration. N. Engl. J. Med. 377, 792-793 (2017).

92. Yoshihara, M., Hayashizaki, Y. \& Murakawa, Y. Genomic instability of iPSCs: challenges towards their clinical applications. Stem Cell Rev. Rep. 13, 7-16 (2017).

93. Cyranoski, D. Japanese man is first to receive 'reprogrammed'stem cells from another person. Nature 10, 1038 (2017).

94. U.K. Moorfields Eye Hospital NHS. Production of iPSC derived RPE cells for transplantation in AMD. https:/clinicaltrials.gov/ct2/show/study/ NCT02464956. Accessed July 21, 2020 (2020).

95. Chakradhar, S. An eye to the future: researchers debate best path for stem cell-derived therapies. Nat. Med. 22, 116-119 (2016)

96. Kaji, K. et al. Virus-free induction of pluripotency and subsequent excision of reprogramming factors. Nature 458, 771-775 (2009).

97. Bracha, P., Moore, N. A. \& Ciulla, T. A. Induced pluripotent stem cell-based therapy for age-related macular degeneration. Expert Opin. Biol. Ther. 17, 1113-1126 (2017).

98. Bakondi, B., Girman, S., Lu, B. \& Wang, S. Multimodal delivery of isogenic mesenchymal stem cells yields synergistic protection from retinal degeneration and vision loss. Stem Cells Transl. Med. 6, 444-457 (2017).

99. Rezanejad, $\mathrm{H}$. et al. In vitro differentiation of adipose-tissue-derived mesenchymal stem cells into neural retinal cells through expression of human PAX6 (5a) gene. Cell Tissue Res. 356, 65-75 (2014).

100. Melief, S. M., Zwaginga, J. J., Fibbe, W. E. \& Roelofs, H. Adipose tissue-derived multipotent stromal cells have a higher immunomodulatory capacity than their bone marrow-derived counterparts. Stem Cells Transl. Med. 2, 455-463 (2013).

101. Siqueira, R. C., Messias, A., Voltarelli, J. C., Scott, I. U. \& Jorge, R. Intravitreal injection of autologous bone marrow-derived mononuclear cells for hereditary retinal dystrophy: a phase I trial. Retina 31, 1207-1214 (2011). 
102. Siqueira, R. C. et al. Resolution of macular oedema associated with retinitis pigmentosa after intravitreal use of autologous BM-derived hematopoietic stem cell transplantation. Bone Marrow Transplant. 48, 612-613 (2013).

103. Siqueira, R. C. et al. Quality of life in patients with retinitis pigmentosa submitted to intravitreal use of bone marrow-derived stem cells (Reticell -clinical trial). Stem Cell Res. Ther. 6, 29 (2015).

104. Park, S. S. et al. Intravitreal autologous bone marrow CD34+ cell therapy for ischemic and degenerative retinal disorders: preliminary phase 1 clinical trial findings. Investig. Ophthalmol. Vis. Sci. 56, 81-89 (2014).

105. Egypt. Al-Azhar University. Safety study of use of autologuous bone marrow derived stem cell in treatment of age related macular degeneration. https:/ clinicaltrials.gov/ct2/show/study/NCT02016508. Accessed July 21, 2020 (2020).

106. Satarian, L. et al. Intravitreal injection of bone marrow mesenchymal stem cells in patients with advanced retinitis pigmentosa; a safety study. J. Ophthalmic Vis. Res. 12, 58-64 (2017).

107. Li, P. et al. Subpopulations of bone marrow mesenchymal stem cells exhibit differential effects in delaying retinal degeneration. Curr. Mol. Med. 16, 567-581 (2016).

108. Sasahara, M. et al. Activation of bone marrow-derived microglia promotes photoreceptor survival in inherited retinal degeneration. Am. J. Pathol. 172, 1693-1703 (2008).

109. Xu, W., Wang, X.-T., Xu, G.-X., Guo, J. \& Huang, L.-B. Stromal cell-derived factor 1a-stimulated mesenchymal stem cells confer enhanced protection against light-induced retinal degeneration in rats. Curr. Eye Res. 39, 69-78 (2014).

110. Yu, B., Zhang, X. \& Li, X. Exosomes derived from mesenchymal stem cells. Int. J. Mol. Sci. 15, 4142-4157 (2014).

111. Park, S. S. et al. Advances in bone marrow stem cell therapy for retinal dysfunction. Prog. Retin Eye Res. 56, 148-165 (2017).

112. Fafian-Labora, J. et al. Influence of age on rat bone-marrow mesenchymal stem cells potential. Sci. Rep. 5, 16765 (2015).

113. Shi, Y. et al. Immunoregulatory mechanisms of mesenchymal stem and stromal cells in inflammatory diseases. Nat. Rev. Nephrol. 14, 493-507 (2018).
114. Lu, B. et al. Human adult bone marrow-derived somatic cells rescue vision in a rodent model of retinal degeneration. Exp. Eye Res. 91, 449-455 (2010).

115. Kalluri, R. \& LeBleu, V. S. The biology, function, and biomedical applications of exosomes. Science 367, 6478 (2020).

116. Xin, $\mathrm{H}$. et al. Systemic administration of exosomes released from mesenchymal stromal cells promote functional recovery and neurovascular plasticity after stroke in rats. J. Cereb. Blood Flow. Metab. 33, 1711-1715 (2013).

117. Xin, H. MicroRNA-17-92 cluster in exosomes enhance neuroplasticity and functional recovery after stroke in rats. Stroke 48, e137 (2017).

118. de Godoy, M. A. et al. Mesenchymal stem cells and cell-derived extracellular vesicles protect hippocampal neurons from oxidative stress and synapse damage induced by amyloid- $\beta$ oligomers. J. Biol. Chem. 293, 1957-1975 (2018).

119. Sun, G. et al. hucMSC derived exosomes promote functional recovery in spinal cord injury mice via attenuating inflammation. Mater. Sci. Eng. C 89 194-204 (2018).

120. Mead, B., Amaral, J. \& Tomarev, S. Mesenchymal stem cell-derived small extracellular vesicles promote neuroprotection in rodent models of glaucoma. Investig. Ophthalmol. Vis. Sci. 59, 702-714 (2018).

121. Moisseiev, E. et al. Protective effect of intravitreal administration of exosomes derived from mesenchymal stem cells on retinal ischemia. Curr. Eye Res. 42 1358-1367 (2017).

122. Safwat, A. et al. Adipose mesenchymal stem cells-derived exosomes attenuate retina degeneration of streptozotocin-induced diabetes in rabbits. J. Circ. Biomark. 7, 1849454418807827 (2018).

123. Zhang, W., Wang, Y. \& Kong, Y. Exosomes derived from mesenchymal stem cells modulate miR-126 to ameliorate hyperglycemia-induced retinal inflammation via targeting HMGB1. Investig. Ophthalmol. Vis. Sci. 60, 294-303 (2019).

124. China. Shanghai General Hospital, Shanghai Jiao Tong University School of Medicine. Role of the serum exosomal miRNA in diabetic retinopathy (DR). https://linicaltrials.gov/ct2/show/NCT03264976. Accessed July 21, 2020 (2020). 